\title{
Androgynous Fasteners for Robotic Structural Assembly
}

\author{
Olivia Formoso, Christine Gregg \\ Greenfield Trinh, Arno Rogg \\ Kenneth Cheung \\ Coded Structures Laboratory \\ NASA Ames Research Center \\ Moffett Field, CA \\ kenny@nasa.gov
}

\begin{abstract}
We describe the design and analysis of an androgynous fastener for autonomous robotic assembly of high performance structures. The design of these fasteners aims to prioritize ease of assembly through simple actuation with large driver positioning tolerance requirements, while producing a reversible mechanical connection with high strength and stiffness per mass. This can be applied to high strength to weight ratio structural systems, such as discrete building block based systems that offer reconfigurability, scalability, and system lifecycle efficiency. Such periodic structures are suitable for navigation and manipulation by relatively small mobile robots. The integration of fasteners, which are lightweight and can be robotically installed, into a high performance robotically managed structural system is of interest to reduce launch energy requirements, enable higher mission adaptivity, and decrease system life-cycle costs.
\end{abstract}

\section{TABle of Contents}

1. INTRODUCTION...$\ldots \ldots \ldots \ldots \ldots \ldots \ldots \ldots \ldots \ldots$

2. Problem Statement $\ldots \ldots \ldots \ldots \ldots \ldots \ldots \ldots \ldots \ldots$

3. Parameterized Design $\ldots \ldots \ldots \ldots \ldots \ldots \ldots \ldots \ldots$

4. Design Analysis And Testing ..............4

5. FinAL DESIGN ..........................

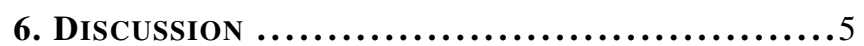

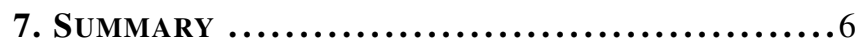

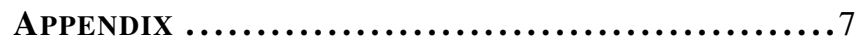

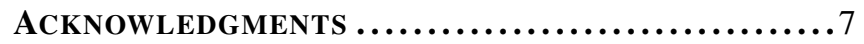

REFERENCES ............................ 7

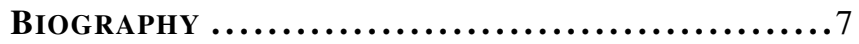

\section{INTRODUCTION}

On-orbit assembly of structures is a long standing technology goal that enables missions reliant on large scale infrastructure, such as space stations, wide aperture transceivers, and exoplanetary habitats. Prior work has demonstrated on-orbit fastening of modular structural components ranging in size and complexity from demonstration trusses [1] to International Space Station modules [2]. For these applications, assembly ConOps complexity and mission adaptivity is seen to benefit from the genderless or androgynous connection mechanisms, meaning that the connected components are copied instances of a single design.

The International Berthing and Docking Mechanism (IBDM) provides an example that includes the ability to work with positional tolerances that are coupled to the capabilities of the Attitude Determination and Control Systems of the components to be connected [2]. At small scales, most

U.S. Government work not protected by U.S. copyright space structure assembly experiments in the literature include an original ConOps that includes an astronaut on an Extra Vehicular Activity (EVA) manipulating parts of the system, with tolerances necessarily befitting human dexterity with EVA gloves [3], [4]. This relationship between the design parameters of fastener systems and the control parameters of intended actuation mechanisms is also studied in modular reconfigurable robotics. Like on-orbit coupling mechanisms, the state of the art in reconfigurable robotics transitioned from gendered designs in early work to androgynous fasteners, and it is widely recognized that the adaptability and robustness of a robot design relies on high misalignment tolerance in the coupling interfaces [5], [6], [7], [8].

Recent efforts to apply robotics and automation to structural systems for aerospace applications [9] are focused on high performance structural systems [10] with relatively simple robots [11]. This provides a requirement for the fastener mechanisms to employ design principles similar to the IBDM in order to relax the positioning requirements of the assembly robots. Unlike self-reconfiguring modular robotics, the structural connection in discrete lattice materials has high structural efficiency requirements, i.e. the stiffness and loading capacity per given mass. For space applications, the ARMADAS robots must construct a structure that is competitive with current state of the art lightweight structures. For this reason, the connection between building-block lattice elements cannot afford self-actuation due to the mass of the associated mechanisms. Instead, the fastening actuation is designed to be provided by the assembling robots.

The mass of the fastening mechanism between building blocks must be minimized, since any nonstructural mass has a parasitic effect on specific properties like modulus and strength, but must maintain geometric features to make robotic fastening reliable with large misalignment tolerances. Because this application calls for actuation that is moved between fasteners, geometric features must ensure not only alignment between fasteners and building-blocks, but also alignment between the fasteners and the robotic driver providing fastener actuation.

\section{Problem Statement}

In this study, we describe a parameterized design of an androgynous captive fastener for autonomous robotic assembly of building block-based lattice structural components. The target system uses cuboctahedron lattice building blocks that are composed of 6 square faces [1] fastened to neighboring blocks via fasteners on the corners of the square face as shown in Figure 1. These fasteners must resist the tensile and shear forces upon loading of the lattice structure to ensure that the struts of the lattice building blocks govern the mechanical behavior of the system (e.g. fail with or before the fasteners). 
What distinguishes this application from reconfigurable robot prior art is the required structural efficiency of the inter-block fastener. The resulting lattice structure must satisfy nearideal specific modulus and strength scaling requirements, which places high structural efficiency requirements on the fastener. Current prototype lattice building blocks are tens of centimeters in characteristic dimension, but the application of the fastener solution presented here should not be understood as constrained to use only at this scale. The same governing mechanics and design processes apply to building block lattices and fasteners of any scale (as long as manufacturing constraints remain proportional or better).
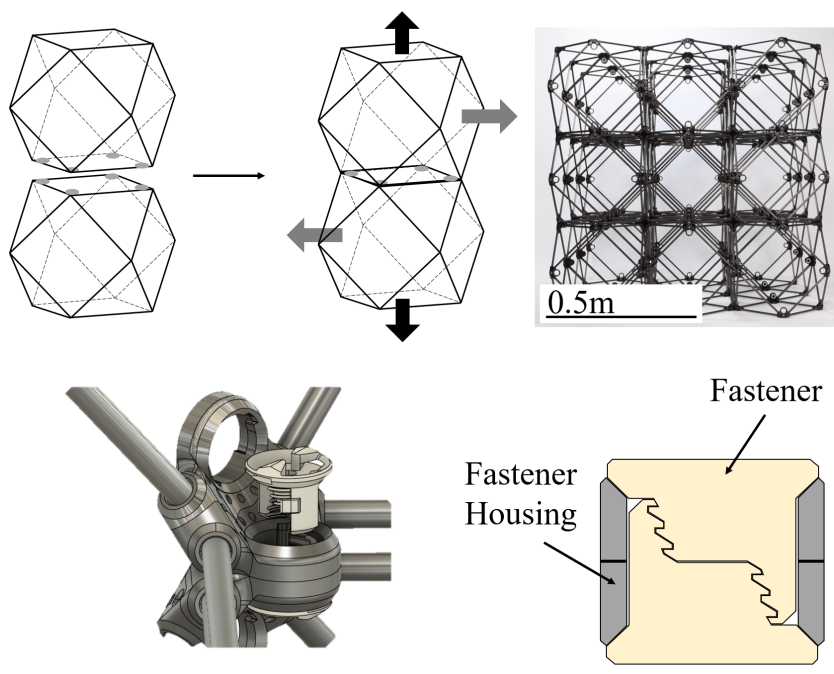

Figure 1. The lattice structure and method of joining can be visualized here: top left - the faces of two cuboctahedron building blocks are connected at their vertices to form the lattice structure with loading in the tensile (black) and shear (gray) directions; top right image of an assembled 3x3x3 lattice structure; bottom left - the fasteners are installed into the fastener housing on a face; bottom right - a section view of fasteners holding together two building block faces.

Additional constraints arise from our need to perform robotic assembly. We estimate that the fasteners should be androgynous, as opposed to traditional male-female fasteners, to remove building-block orientation requirements and allow for assembly in all orthogonal build directions. We also require fasteners to be captive in the building block structural elements to reduce the logistical complexity of needing to carry additional fasteners for assembly. The fasteners must provide a mechanically reversible connection to allow reconfiguration [12]. We also wish to prioritize ease of robotic activation with low activation force and high holding strength, as well as low robotic motion complexity (low degrees of freedom with a low number of states being desired).

A wide range of fastener types were considered, including bayonet connectors, shear pins (inserted orthogonal to the net tensile loading directions), press and interference fit connectors, as well as conventional nuts and bolts. Our requirement for androgyny limited consideration of the latter to comparison and benchmarking. The use of a screw mechanism to achieve activation/holding force asymmetry using rotational activation of tensile preload is noted as a positive attribute of these and bayonet connectors. Due to geometry of the structural system joints, a rotationally activated tensile fastener based on the principles of a bolted connection was deemed more feasible than the shear pin based design. The basic geometry and operation of the fastener design can be seen in Figures 2 and 3.
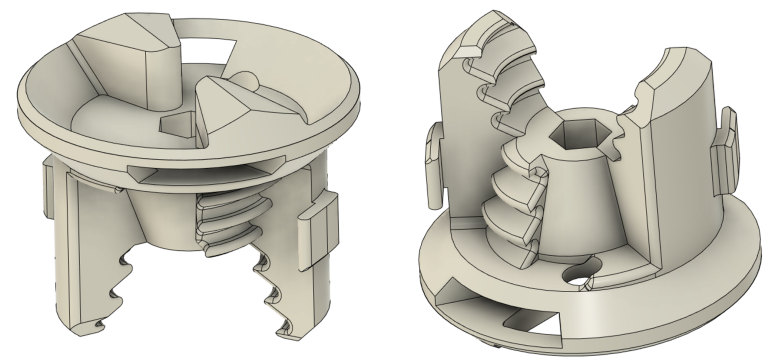

Figure 2. The target fastener design is captive and androgynous to limit the complexity of the assembly system. A rotationally activated tensile fastener was chosen to decouple the activation and holding force, relaxing the requirements of the robotic motion.

\section{Geometric Problem Statement}

The fastener must meet strength requirements while minimizing mass. Structural requirements arise not only from operational axial tensile and shear loads, but also loads arising during torquing and installation.

The fastener must also make robotic assembly as easy as possible, which we define as allowing successful fastener activation with the lowest required positioning precision of the robotic driver and the lowest driving torque for a given fastener strength. The precision requirements of the robotic driver can be decomposed into three fastener design metrics: positional tolerance, rotational tolerance, and driver azimuth angle tolerance. These are calculated based on the geometry of the features in the fastener design.

Positional Tolerance-We define positional tolerance as the $\mathrm{X}$ and $\mathrm{Y}$ limits (or radial limits) that allow the driver bit to be centered onto the fastener when an axial force is applied. It describes the envelope over which the fastener can guarantee centering the driver during fastener activation. We wish to maximize the positional tolerance to lower the required precision of robotic driver positioning to guarantee successful fastener activation.

Rotational Tolerance-We define rotational tolerance as the maximum rotational angle offset that allows the fastener and driver bit to engage fully when axial force is applied.

Driver Azimuth Angle Tolerance-The maximum offset angle $(\theta)$ of the driver bit from the orthogonal position in which the driver bit and the fastener are still engaged when rotating. We wish to maximize the driver azimuth angle tolerance to relax positioning requirements of the robotic driver.

\section{Parameterized Design}

For the defined problem statement, two main features of a rotationally activated fastener affect the mechanical strength and robotic ease of assembly: the fastener head and the fastener thread. Analogous to the head and thread of a traditional bolt, the head design defines the interface to the surfaces to be joined and specifies the interface and tolerance requirements of the robotic driver. The thread section transfers the load from one fastener to another (as the thread of a traditional bolt would transfer load to the nut) (Figure 1). Design parameters 

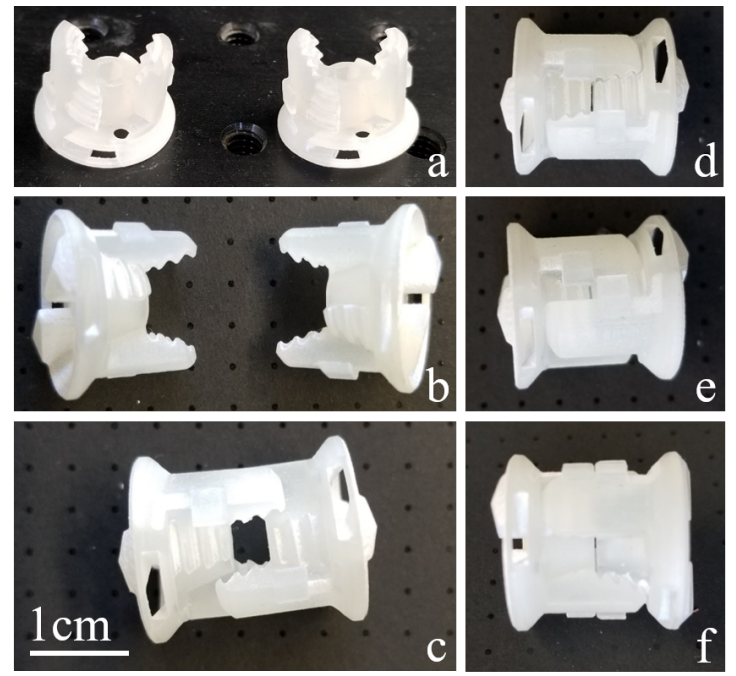

Figure 3. The fasteners are joined as follows: (a) two androgynous fasteners, (b) fasteners are placed in opposing faces of the building blocks to be assembled (blocks not shown here), (c) the fasteners are pushed together axially and held in place using alignment features, (d) the fasteners are fully inserted in the axial direction, (e) the fasteners are rotated clockwise to engage the threads, (f) the fasteners hit a stop as they reach their maximum rotation and are fully engaged. Fasteners shown here are 3D printed prototypes, but the final design can be injection molded or machined.

for both the head and thread were established to evaluate the performance of a fastener design for a given application. For this study, the head and thread section are defined as separate entities and are developed independently of each other. After independent parameterization, promising head and thread combinations were simulated and tested as unified structures to ensure that the interface between the head and thread was not the point of failure.

\section{Thread}

The function of the threads is to create an interface that will transfer tensile load between two components. As with a traditional screw, the friction between the interlocking threads compresses the two parts together and through elastic deformation provides a pre-loaded joint. The following parameters are used to characterize the thread design and are shown in Figure 4.

Thread Angle-We define the thread angle as the angle between adjacent thread surfaces. Standard types of screw threads are taken into consideration including V, American National, British Standard, square, Acme, buttress, and knuckle. [13]

Number of Threads-We define this as the total number of threads along the length of the fastener.

Pitch-We define the pitch of the threads to be the axial distance between the crests of adjacent threads.

Taper-The thread taper is defined as the complementary angle of the right circular cone whose center axis coincides with the center of the fastener and passes through the crests of the threads.

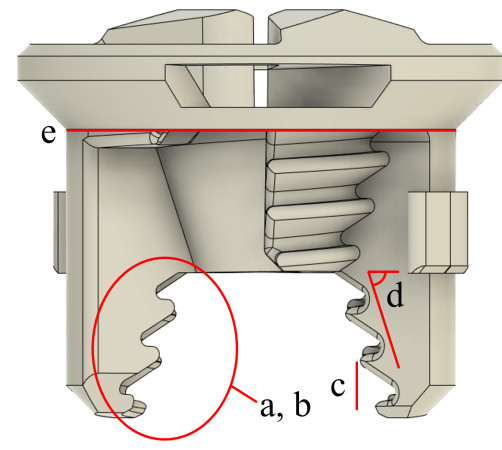

Figure 4. The fastener parameters used to characterize the threads can be visualized here: (a) thread angle, (b) number of threads, (c) pitch, (d) taper angle, and (e) external diameter.

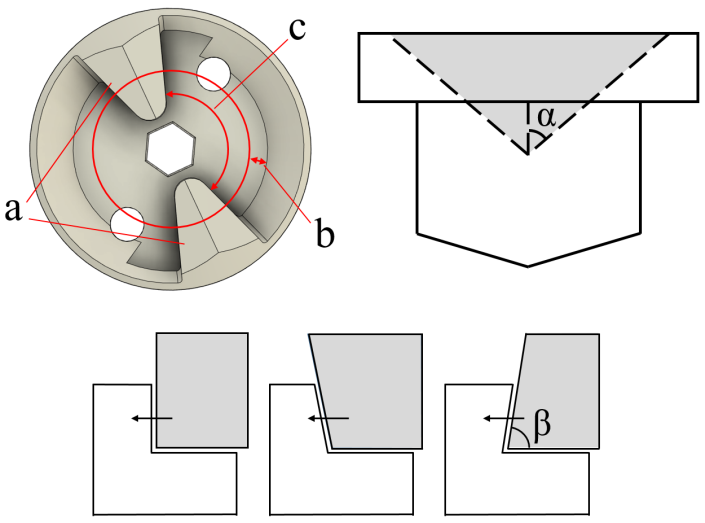

Figure 5. The fastener parameters used to characterize the head can be visualized here: top left - (a) number of teeth, (b) radial fit, (c) rotational fit; top right - alignment feature; bottom - draft angle.

External Diameter-We define this as the maximum diameter of the overall thread features.

Material Properties-The absolute strength and performance of the threads is dependent on the constituent material of the fastener.

\section{Head}

The head design is integral to promoting ease of robotic assembly and transfer of torque to engage the fastener. While there are various traditional screw head types, the majority are designed for human use where a consistent axial force is applied during the fastening motion. We desire to explore unconventional designs more suitable for robotic assembly and utilize the following parameters to characterize the head design, shown in Figure 5.

Number of Teeth-We define the number of teeth as the number of points of contact between the driver bit and fastener.

Alignment Features - To radially align the driver bit to the fastener head, we find that a cone performs better than the other options considered.

Driver Fit-We define the driver fit as the tolerance between the fastener and driver bit features. This can be decomposed into the radial fit and rotational fit. 
Draft Angle-The draft angle is defined as the angle between the driving surface of the teeth and the fastener head plane.

Material Properties-As with the threads, the strength of the material will depend on the constituent material. The head and threads will be fabricated out of the same material.

\section{Design Analysis and Testing}

To better understand how the parameters outlined in the previous section affect our desired metrics, we explored different areas of the solution space by considering both traditional and custom designs. These initial prototypes were tested and evaluated both quantitatively and qualitatively as a first step in determining a final design for our system.
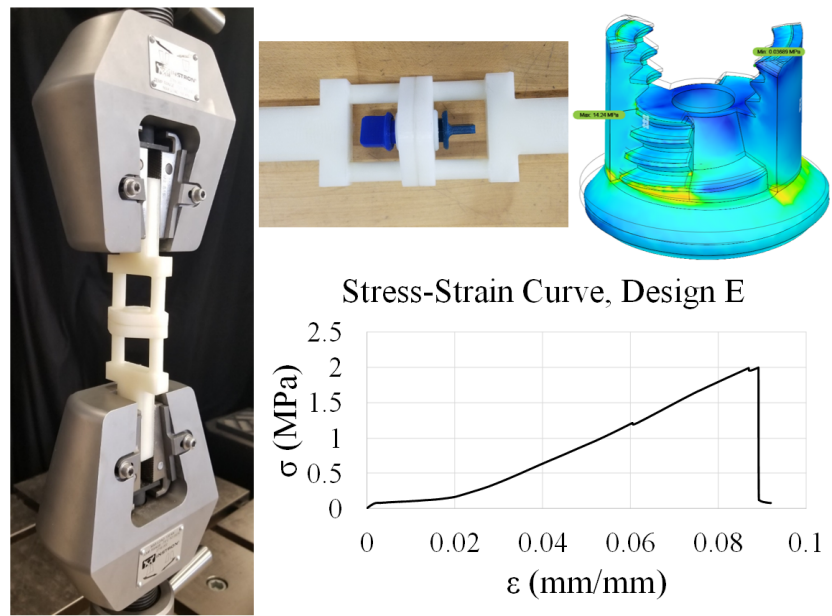

Figure 6. Tensile testing was performed to assess fastener performance: top middle - two fasteners are aligned axially in the test rig and turned fully to fasten; left - the rig is placed into a tensile testing machine; bottom right - a typical stress strain curve for the

fastener specimen. The highest peak is recorded to calculate the maximum load; top right - a typical result of an FEA analysis on a fastener design.

Five different versions of fastener threads were designed to examine the relationship between the parameters and our metrics. The designs are outlined in Table 1 and a subset are shown in Figure 8. To compare the structural performance of the different thread designs, tensile testing was performed on $3 \mathrm{D}$ printed versions of the fasteners to determine their relative load capacities. Because the faces of the building blocks contain hirth joints for alignment during assembly, these features take most of the shear loads; therefore, the primary loading on the fastener should be tensile. Test fixtures (Figure 6) simulated the housing constraints and joint thickness of the joined lattice faces while providing gripping surfaces for the grips of the mechanical testing machine.

Five specimens of each type of fastener were tested during this experiment. The assembly was loaded axially until failure, and the peak load during the test was recorded. The average peak load per mass and standard deviation data can be seen in Figure 7 and Table 1. During testing, it was observed that the fasteners would either break at the interface of the head and threads or at the middle of the threads. Thread designs $\mathrm{A}, \mathrm{B}$, and $\mathrm{E}$ broke at the middle of the threads, while thread designs $\mathrm{C}$ and $\mathrm{D}$ broke at both the middle of the threads and at the head and thread interface. The peak loads for a specific thread design in these instances did not show significant differences, thus the experimental performance of the specimens were still viable. This same behavior can be seen in the image of the FEA simulation in Figure 6, validating our FEA simulation parameters and constraints. This allows the simulation setup to be used to evaluate alternative fastener designs.

\section{Experimental and Simulated Load Capacity of Thread Designs}

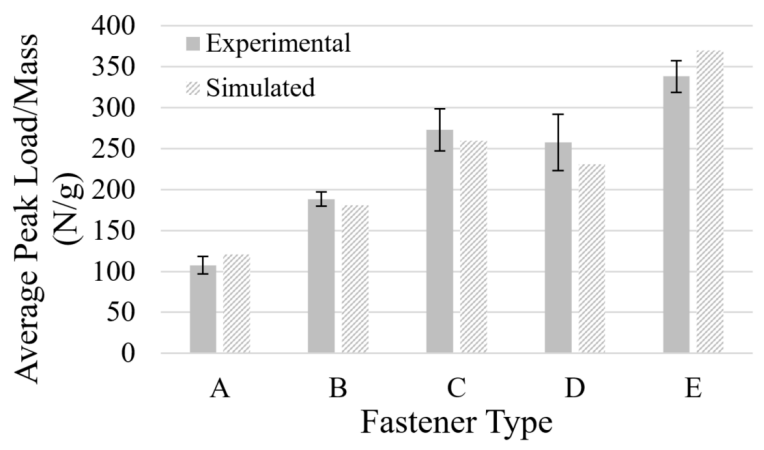

Figure 7. Various combinations of thread parameters were designed to understand the effects of changing parameters. A tensile test was conducted on $3 D$ printed models to compare load capacity of the different specimens. Standard deviation bars are added to the experimental results.

Comparing designs $\mathrm{A}$ and $\mathrm{B}$, it is clear that the strength of the fastener increases with a higher number of threads. Based on the ratio of load bearing on a traditional screw, we set a thread taper of $70^{\circ}$ for design $C$ to attempt a uniform load distribution across the threads and minimize parasitic mass [14]. Comparing this to design D which has a $40^{\circ}$ taper, we see a slight decrease in strength with a smaller taper angle. A taper angle of $90^{\circ}$ is not physically possible, and a taper of $0^{\circ}$ is similar to a traditional screw. Changing the thread angle of the fastener shows a large effect on the strength. Fastener $\mathrm{D}$ has a $60^{\circ}$ thread angle, corresponding to V-shaped threads. Modifying the thread angle to $30^{\circ}$, creating a buttress style thread, increased the strength of the fastener significantly.

A variety of head designs were considered to determine the range of tolerances that are traditionally seen in fasteners. Known head designs such as phillips, flat, and hex configurations were analyzed, as well as more uncommon styles such as the space dock and tool changer design. A subset of the analyzed designs are shown in Figure 8 and tolerance metrics summarized in Table 2.

The tolerances for the head designs are largely dependent on the fit of the corresponding driver. The transverse and rotational tolerances of standard head designs, such as the phillips and flat, are much smaller than a custom version. The rotational tolerances of the square, hex, and torx heads are much higher than the other geometries; however, they are based off the angle of the alignment cone that encompasses the head and not on the head design itself. Based on the data for the tool changer, a higher number of teeth will limit the potential rotational tolerance of the design. 
Table 1. Fastener Thread Parameters and Load Capacities

\begin{tabular}{|c|c|c|c|c|c|c|}
\hline Design & Thread Angle & No. of Threads & Pitch, mm & Taper & $\begin{array}{c}\text { Max Load } \text { avg }_{\text {Mass, N/g }} \text { per } \\
\text { Max Load }_{\text {sim }} \text { per } \\
\text { Mass, N/g }^{\text {Mer }}\end{array}$ \\
\hline A & $0^{\circ}$ & 2 & 0.25 & $0^{\circ}$ & 107.4 & 121.1 \\
B & $0^{\circ}$ & 4 & 0.25 & $0^{\circ}$ & 188.3 & 181.1 \\
C & $60^{\circ}$ & 6 & 1.90 & $70^{\circ}$ & 272.9 & 259.4 \\
D & $60^{\circ}$ & 6 & 1.90 & $40^{\circ}$ & 257.4 & 231.2 \\
E & $30^{\circ}$ & 6 & 1.90 & $70^{\circ}$ & 338.3 & 369.7 \\
\hline
\end{tabular}

Table 2. Tolerance Metrics for Various Head Geometries

\begin{tabular}{|c|c|c|c|c|}
\hline Head Type & Transverse (x-dir), mm & Transverse (y-dir), mm & Rotational & Drive Angle \\
\hline Phillips & 1.0 & 1.0 & $2.5^{\circ}$ & $15^{\circ}$ \\
Flat & 1.0 & 7.6 & $3.5^{\circ}$ & $10^{\circ}$ \\
Square & 0.9 & 0.9 & $45^{\circ}$ & $20^{\circ}$ \\
Hex & 1.0 & 1.0 & $30^{\circ}$ & $20^{\circ}$ \\
Torx & 1.4 & 1.4 & $30^{\circ}$ & $20^{\circ}$ \\
Space Dock & 1.5 & 1.5 & $10^{\circ}$ & $2^{\circ}$ \\
Tool Changer & 6.0 & 6.0 & $3^{\circ}$ & $4^{\circ}$ \\
\hline
\end{tabular}

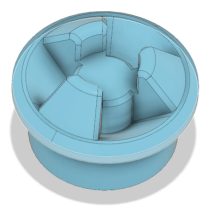

Space Dock

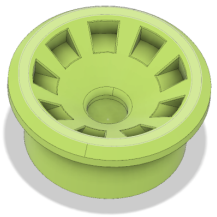

Tool Changer

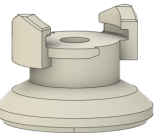

Thread Design A

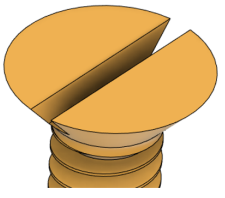

Flat

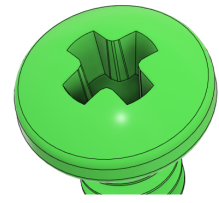

Phillips

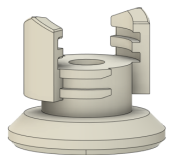

Thread Design B

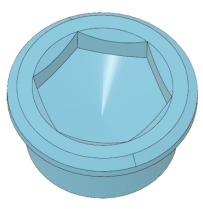

Hex

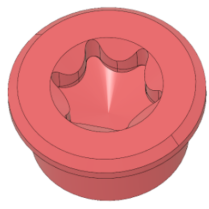

Torx

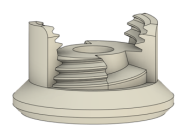

Thread Design D
Figure 8. Several fastener head geometries were evaluated, shown in the top two rows, including some COTS examples and designs inspired by similar robotic applications. Three of the thread designs are shown in the bottom row to visualize the variety of designs.

\section{Final Design}

Our final thread design prioritizes the strength of the connection. For the screw thread, we utilize a sawtooth buttress style thread, which has a smaller thread angle, $30^{\circ}$, than a traditional buttress, $45^{\circ}$, allowing a higher surface area of contact. The faces in contact are perpendicular to the direction of the axial forces, providing a higher load capacity. The shape of the teeth also promotes less friction than other thread shapes, which requires less torque to engage and promotes ease of robotic actuation.
The external diameter is set to the maximum allowable based on the unit cell geometry, $15.9 \mathrm{~mm}$, to provide a maximum contact area. The number of threads, six, and pitch, 1.9 $\mathrm{mm}$, were determined by fitting as many threads within the allowable height of the fastener, $15.9 \mathrm{~mm}$, set by unit cell geometry and the design specifications of a sawtooth buttress style thread profile. In general, increased thread number distributes the load across more surfaces and prevents the possibility of the shear failure on a single thread. However, the number of threads that fit is constrained by the general rule of thumb for thread engagement that states the thread engagement should be approximately equal to the diameter of the threads.

The head design prioritizes ease of robotic assembly. The two tooth design maximizes the spacing between the teeth to provide greater rotational tolerances for robotic driver engagement. The radial driver fit was chosen to have a spacing of $3.0 \mathrm{~mm}$ and the rotational fit was minimized to allow for a large error in the rotational position of the driver. The alignment cone is also maximized at $45^{\circ}$ to allow a greater radial tolerance. The draft angle was set at $90^{\circ}$ to promote simple controls for the robotic end effector and not require any extra steps for engagement and disengagement. The driver azimuth angle is driven by the height of the teeth, and this is maximized to allow for the largest tooth height possible without any interference between the features when the fasteners are in the unengaged captive position.

The final fastener parameters and performance characteristics are summarized in Table 3 and the design is shown in Figure 2 .

\section{Discussion}

The weighting of the metrics for evaluating the fastener design are dependent on the specific system requirements and applications. The parameters discussed in this study are found to significantly affect mechanical performance and robotic assembly. 
Table 3. Case Study Parameter Values and Final Metrics

\begin{tabular}{|c|c|}
\hline Fastener Parameter & Value \\
\hline Thread Angle & $30^{\circ}$ \\
Number of Threads & 3 \\
Pitch & $1.9 \mathrm{~mm}$ \\
Taper & $70^{\circ}$ \\
External Diameter & $15.9 \mathrm{~mm}$ \\
Number of Teeth & 2 \\
Alignment Features & $45^{\circ}$ \\
Radial Fit & $3.0 \mathrm{~mm}$ \\
Rotational Fit & $50.4^{\circ}$ \\
Draft angle & $90^{\circ}$ \\
\hline Performance Metric & Value \\
\hline Transverse Tolerance & $3.0 \mathrm{~mm}$ \\
Rotational Tolerance & $50.4^{\circ}$ \\
Driver Azimuth Angle Tolerance & $16.3^{\circ}$ \\
\hline
\end{tabular}

Focusing first on the thread design, the majority of the parameters have direct effects on the strength of the fastener and connection. The thread angle is studied based on standard screw types. Standard V-threads are widely used in industry and are usually suitable for holding components together under conventional loads. For higher and unidirectional loads, a buttress thread is more suitable as the face of the thread that is bearing the load is perpendicular to the load direction. Acme and square threads are mainly used for power transmission, and based on their larger profile may not be as mass efficient as the previous options. Also worth consideration is that increasing the thread angle will increase the friction between mating parts and lead to more wear of the screw, which will drive up torque requirements for the assembler.

A higher number of teeth can provide a larger total surface for load bearing, which can increase the strength of the fastener. However, this can lead to a higher chance of crossthreading when two fasteners are engaged. The pitch of the fastener also depends on the thread profile and length of the fastener. Depending on the application, a finer pitch may be necessary for smaller travel distances or reducing the torque requirements; however, studies have shown that a coarser pitch displays a higher fatigue life [15].

The tapering of threads is usually implemented in industry to create an airtight seal for rigidity in pipe fittings. We look at the thread taper as a way to minimize the mass of the fastener [16]. Finally, increasing the external diameter of the threads provides a larger area of contact and thus increases the load capacity of the fastener.

Looking at the head design, we attempt to base a design off a traditional fastener head while prioritizing ease of robotic assembly. The basic alignment feature for the driver is a cone. The angle of the cone will depend on the axial force from the end effector and the friction between the materials to maximize the allowable positional tolerances. The driver fit affects transversal, rotational, and entry angle tolerance. It is ideal to maximize this effect while ensuring the driver is still able to engage and turn the fastener. The draft angle helps to align the driver to the fastener head. An angle greater than $90^{\circ}$ can cause the driver to cam out during fastening, while an angle less than $90^{\circ}$ can reduce this effect and promote engagement of the driver with the head of the fastener. For this instance, the driver will have to be backdriven in order to provide clearance to disengage.

The number of teeth affects the transfer of torque and the ease of driver engagement. A lower number of teeth allows for more space between teeth for alignment features, but the smaller area of contact leads to inefficient torque transfer. On the other hand, a higher number of teeth provides a better force distribution and transfer of torque, but leads to higher positioning requirements for an end-effector. For high-torque applications, a two-tooth head design may be inappropriate due to yielding of material at the teeth or mass-inefficient geometry to avoid yield.

Finally, all designs must remain cognizant of constituent material, manufacturability, and costs. Due to the high number of fasteners needed, our application required that the design be injection-moldable, which constrained geometric features and material availability.

The current design relies on friction to resist coming apart (i.e. prevent rotation). To qualify these for use in space applications, a secondary locking feature should be integrated to prevent the fasteners from backing out. One solution is to add a hole that spans both fasteners in the engaged position and insert a locking pin after the fasteners are tightened.

\section{SUMMARY}

On orbit assembly of space structures is critical to enable missions that demand large scale infrastructure. The future of space construction will require robots to operate autonomously in extreme environments. Utilizing traditional fastening components results in complex assembly requirements and intricate robotic systems with multiple points of failure. To create a fastener that achieves both the strength requirements and robotic assembly specifications for any system, we develop a parameterized model that can be tuned for such an application.

We focus on metrics that demonstrate ease of robotic assembly and the desired structural performance. Deconstructing the thread and head design into their constituent parameters allows us to fine tune a design based on the limitations of a system. The metrics evaluated in this study include the positional tolerance, rotational tolerance, driver angle tolerance, and the load capacity of the fastener. Exploring specific instances throughout the solution space allowed us to create a fastener design that achieves a high load capacity and large engagement tolerances for a robotic end-effector.

Future extensions of this work includes analysis and optimization of other types of joining methods, e.g. shear pins, latches, or snap fit joints. This initial study focuses on a joining area that is relatively small compared to the overall size of the unit cell building block, and a future study would envision how these joining methods evolve as the building block increases in size. The work described in this paper provides an effort to understand and characterize complex interactions between robotic assembly systems and fastening hardware. 


\section{APPENDIX}

\section{ACKNOWLEDGMENTS}

The authors thank the NASA STMD Game Changing Development (GCD) Program for support (Automated Reconfigurable Mission Adaptive Digital Assembly Systems (ARMADAS) Project).

\section{REFERENCES}

[1] W. R. Doggett, J. Dorsey, J. Teter, D. Paddock, T. Jones, E. E. Komendera, L. Bowman, C. Taylor, and M. Mikulas, "Persistent Assets in Zero-G and on Planetary Surfaces: Enabled by Modular Technology and Robotic Operations," 2018 AIAA SPACE and Astronautics Forum and Exposition, no. September, pp. 1-33, 2018. [Online]. Available: https://arc.aiaa.org/doi/10.2514/6.2018-5305

[2] J. Cook, V. Aksamentov, T. Hoffman, and W. Bruner, "ISS interface mechanisms and their heritage," AIAA SPACE Conference and Exposition 2011, 2011.

[3] I. Bekey, "Space construction results: The EASE/ACCESS flight experiment," Acta Astronautica, vol. 17, no. 9, pp. 987-996, 1988.

[4] J. J. Watson, T. J. Collins, and H. G. Bush, "A history of astronaut construction of large space structures at NASA Langley Research Center," in Aerospace Conference Proceedings, 2002. IEEE, vol. 7, 2002, pp. 73569-7-3587 vol.7.

[5] W. Saab, P. Racioppo, and P. Ben-Tzvi, "A review of coupling mechanism designs for modular reconfigurable robots," Robotica, vol. 37, no. 2, pp. 378-403, 2019.

[6] C. Parrott, T. J. Dodd, and R. Groß, "HiGen: A highspeed genderless mechanical connection mechanism with single-sided disconnect for self-reconfigurable modular robots," IEEE International Conference on Intelligent Robots and Systems, no. Iros, pp. 3926-3932, 2014.

[7] W. Saab and P. Ben-Tzvi, "A genderless coupling mechanism with six-degrees-of-freedom misalignment capability for modular self-reconfigurable robots," Journal of Mechanisms and Robotics, vol. 8, no. 6, pp. 1-9, 2016.

[8] S. G. M. Hossain, C. A. Nelson, and P. Dasgupta, "ROGENSID- A Rotary Plate Genderless Single-Sided Docking Mechanism for Modular Self-Reconfigurable Robots," in Proceedings of the ASME 2013 International Design Engineering Technical Conferences and Computers and Information in Engineering Conference, Portland, Oregon, 2013, pp. 1-7.

[9] "Automated Reconfigurable Mission Adaptive Digital Assembly Systems (ARMADAS)," 2019. [Online]. Available: https://gameon.nasa.gov/projects/automatedreconfigurable-mission-adaptive-digital-assemblysystems-armadas/

[10] C. E. Gregg, J. H. Kim, and K. C. Cheung, "Ultra-light and scalable composite lattice materials," Advanced Engineering Materials, vol. 1800213, pp. 1-6, 2018.

[11] B. Jenett and K. Cheung, "Bill-e: Robotic platform for locomotion and manipulation of lightweight space structures," in 25th AIAA/AHS Adaptive Structures Conference, 2017, p. 1876.

[12] C. E. Gregg, B. Jenett, and K. C. Cheung, "Assembled, modular hardware architectures-what price reconfigurability?" in 2019 IEEE Aerospace Conference. IEEE, 2019, pp. 1-10.

[13] E. Schwartz and J. H. Bickford, "Screw threads," Handbook of Bolts and Bolted Joints, p. 121, 1998.

[14] "Screw thread design." [Online]. Available: https://www.fastenal.com/en/78/screw-thread-design

[15] G. Majzoobi, G. Farrahi, and N. Habibi, "Experimental evaluation of the effect of thread pitch on fatigue life of bolts," International journal of fatigue, vol. 27 , no. 2 , pp. 189-196, 2005.

[16] D. Zhang, S. Gao, S. Niu, and H. Liu, "A prediction method for load distribution in threaded connections," Journal of Theoretical and Applied Mechanics, vol. 56, 2018.

\section{BIOGRAPHY}

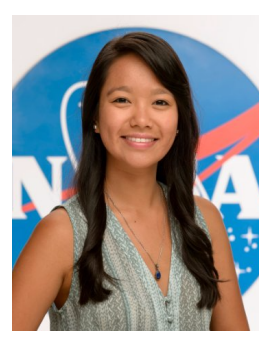

Olivia Formoso received her B.S. degree in Chemical Engineering from the University of Florida in 2016. Currently, she is a research engineer at the Coded Structures Lab at NASA Ames Research Center and is pursuing her M.S. in Mechanical Engineering at San Jose State University. Her research is focused on digital material structures and robotics.

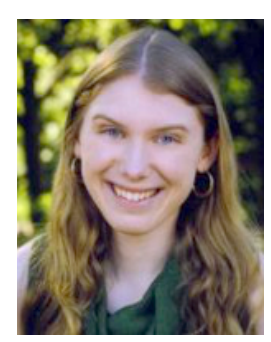

Christine Gregg received her Ph.D. from the Department of Mechanical Engineering at UC Berkeley, where she was a NASA Space Technology Research Fellow. Her thesis focused on digital lattice structures and lattice fracture mechanics. She works in the ARC Coded Structures Laboratory (CSL).

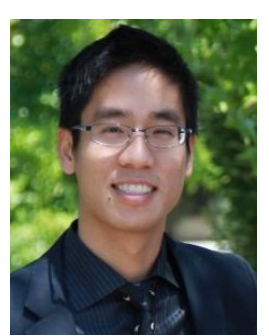

Greenfield Trinh is a research engineer in the Coded Structures Lab at NASA Ames Research Center. His current research activities include automated assembly of digital material structures and robotics. He received his B.S. in Physics from UC Riverside and M.S. in Aerospace Engineering from San Jose State University. 


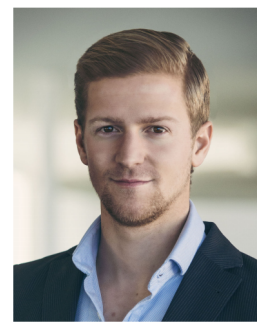

Arno $\boldsymbol{R o g g}$ received his M.S. in microengineering from the Swiss Institute of Technology of Lausanne (EPFL). He is currently working in the Intelligent Robotics Group at NASA Ames Research Center. His work focuses on planetary rover research and development with a focus on rover mobility in difficult terrains and high reliability systems integration.

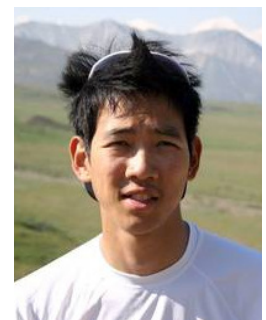

Kenneth Cheung received his Ph.D. from the Center for Bits and Atoms at the Massachusetts Institute of Technology. He helps to run the NASA ARC Coded Structures Laboratory (CSL), which conducts research on the application of building block based materials and algorithms to aeronautical and space systems. As a member of the NASA ARC Intelligent Systems Division and affiliate of the office of the Center Chief Technologist, he serves as a technical lead on advanced materials and manufacturing. 